\title{
The preliminary study of the endovascular management of brain arteriovenous malformations by ethanol sclerotherapy
}

\author{
Yingkun He,MD; Weixing Bai,MD; Tianxiao Li,MD,PhD;
}

\author{
Cerebrovascular Division, Department of Interventional Center, \\ Henan Provincial People's Hospital, Zhengzhou University , \\ No. 7, Weiwu Road, Zhengzhou, 450000, China \\ E-mail: heyingkun@vip.126.com
}

Purpose: To discuss the safety of ethanol ablation therapy in treating brain arteriovenous malformation (bAVM)

Materials \& Methods: Between September 3, 2018 and November 11,2018, a total of 12 patients with bAVM received targeted endovascular ethanol ablation therapy.

Results: Ethanol ablation therapy was carried out on 19 arteries in 12 patients. A total of 13 target aneurysms and 6 target arteriovenous fistulae were ablated. Among the 19 target arteries, 15 were completely ablated by pure ethanol injection, 2 were cured by ethanol combined with Onxy glue injection, one was cured by ethanol injection of arteriovenous fistula together with transvenous embolization, and one was demonstrated mild contrast opacification within the target aneurysm after treatment. Eight of the 12 patients developed different degrees of brain edema.

Five of the 8 patients had newly-developed cerebral infarction.

Only one patient remained obvious neurological impairment at the time of discharge.

Conclusions: For the treatment of bAVM, endovascular ethanol ablation maybe technically feasible and clinically safe, although studies with large sample are still needed to further clarify its effect .
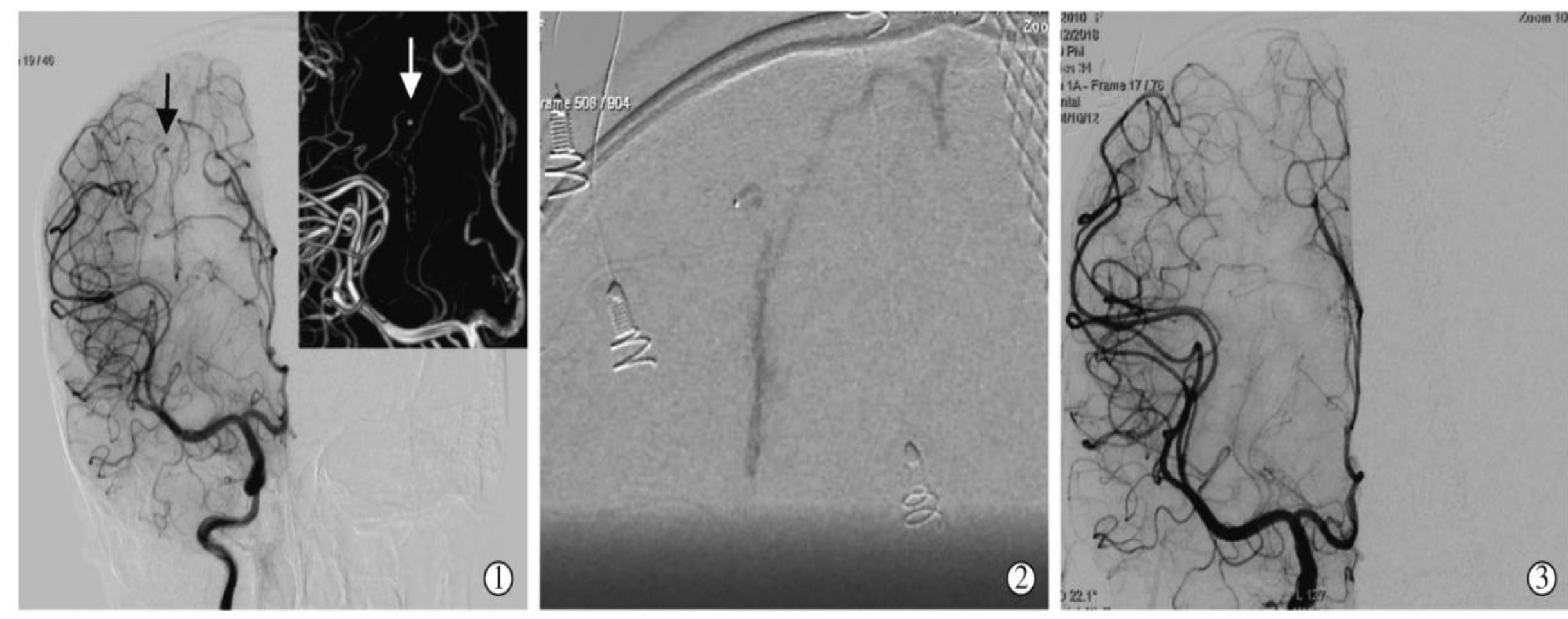

(1) Right internal carotid artery digital subtraction angiography (DSA) showed the nidus was located on the right occipital lobe and an aneurysm on the feeding artery (arrow). (2)After the microcatheter was in position, the rapid bolus injection of the mixture of absolute ethanol and $60 \%$ of iohexol 320 was performed. (3)The postoperative DSA showed the target aneurysm was disappear, and few residues were found. Three-month

angiography follow-up confirms the complete occlusion of the residual AVM

\begin{tabular}{|c|c|c|c|c|c|c|c|c|c|c|c|c|}
\hline \multirow{2}{*}{ Patient } & \multirow{2}{*}{$\begin{array}{l}\text { Gender } \\
\text { /Age }\end{array}$} & \multirow{2}{*}{$\begin{array}{l}\text { Scale } \\
*\end{array}$} & \multirow{2}{*}{ Location } & \multirow{2}{*}{$\begin{array}{l}\text { Hemorrhage } \\
\text { Type }\end{array}$} & \multirow{2}{*}{$\begin{array}{l}\text { Provoc- } \\
\text { ation } \\
\text { Test }\end{array}$} & \multirow{2}{*}{$\begin{array}{l}\text { Number } \\
\text { Targets }\end{array}$} & \multirow{2}{*}{$\begin{array}{l}\text { Ethanol } \\
/ \mathrm{ml}\end{array}$} & \multirow{2}{*}{$\begin{array}{l}\text { Completely } \\
\text { Ablation or } \\
\text { Not }\end{array}$} & \multirow{2}{*}{$\begin{array}{l}\text { New-Onset } \\
\text { Cerebral Edema/ } \\
\text { Infarction } \\
\text { Postoperatively }\end{array}$} & \multicolumn{3}{|c|}{ NIHSS Score } \\
\hline & & & & & & & & & & Preoperative & $\begin{array}{lr}2 & \text { Days } \\
\text { Postoperatively }\end{array}$ & Discharge \\
\hline 1 & $\mathrm{M} / 28$ & IV & $\begin{array}{l}\text { Left Temporal } \\
\text { Insula }\end{array}$ & Parenchyma & / & 1 Aneurysm & 2.3 & Yes & $\mathrm{No} / \mathrm{No}$ & 4 & 4 & 2 \\
\hline 2 & $F / 26$ & II & $\begin{array}{l}\text { Top of The } \\
\text { Corpus } \\
\text { Callosum }\end{array}$ & $\begin{array}{l}\text { Parenchyma, } \\
\text { Ventricle }\end{array}$ & l & 2 Aneurysms & 13.6 & Yes & Yes/No & 0 & 0 & 0 \\
\hline 3 & $\mathrm{~F} / 8$ & II & $\begin{array}{l}\text { Right Parietal- } \\
\text { Occipital Area }\end{array}$ & Parenchyma & - & 1 Aneurysm & 5.6 & Yes & Yes/Yes & 0 & 0 & 0 \\
\hline 4 & $\mathrm{M} / 8$ & III & Diencephalon & $\begin{array}{l}\text { Parenchyma, } \\
\text { Ventricle }\end{array}$ & + & 1 Aneurysm & 3.0 & Yes & Yes/Yes & 0 & 5 & 2 \\
\hline 5 & $F / 16$ & III & Right Thalamus & Ventricle & l & $\begin{array}{l}1 \text { Aneurysm, } \\
1 \text { Fistula }\end{array}$ & 10.2 & No & $\mathrm{No} / \mathrm{No}$ & 0 & 0 & 0 \\
\hline 6 & $F / 26$ & IV & $\begin{array}{l}\text { Left } \\
\text { Frontotemporal } \\
\text { Area }\end{array}$ & $\begin{array}{l}\text { No } \\
\text { Hemorrhage }\end{array}$ & - & $\begin{array}{l}1 \text { Aneurysm, } \\
3 \text { Fistulas }\end{array}$ & 8.0 & Yes & $\mathrm{No} / \mathrm{No}$ & 0 & 0 & 0 \\
\hline 7 & $\mathrm{M} / 73$ & III & Right Thalamus & $\begin{array}{l}\text { No } \\
\text { Hemorrhage }\end{array}$ & - & 1 Aneurysm & 7.7 & Yes & Yes/Yes & 0 & 2 & 0 \\
\hline 8 & $\mathrm{~F} / 7$ & II & $\begin{array}{l}\text { Left Parietal- } \\
\text { Occipital Area }\end{array}$ & Ventricle & - & $\begin{array}{l}1 \text { Aneurysm, } \\
1 \text { Fistula }\end{array}$ & 6.0 & Yes & Yes/No & 0 & 0 & 0 \\
\hline 9 & $F / 16$ & III & $\begin{array}{l}\text { Left Frontal- } \\
\text { Parietal Area } \\
\text { Left }\end{array}$ & Parenchyma & l & 1 Fistula & 3.0 & Yes & Yes/Yes & 0 & 2 & 2 \\
\hline 10 & $\mathrm{M} / 18$ & IV & $\begin{array}{l}\text { Frontotemporal, } \\
\text { Basal Ganglia } \\
\text { Area }\end{array}$ & $\begin{array}{l}\text { Parenchyma, } \\
\text { Ventricle }\end{array}$ & / & $\begin{array}{l}1 \text { Aneurysm, } \\
1 \text { Fistula }\end{array}$ & 11.2 & Yes & $\mathrm{No} / \mathrm{No}$ & 31 & 31 & 31 \\
\hline 11 & $M / 23$ & I & $\begin{array}{l}\text { The Right } \\
\text { Temporal Area }\end{array}$ & Parenchyma & - & 1 Fistula & 7.6 & Yes & Yes/Yes & 0 & 0 & 0 \\
\hline 12 & $F / 26$ & IV & Left Thalamus & $\begin{array}{l}\text { Parenchyma, } \\
\text { Ventricle, } \\
\text { SAH }\end{array}$ & I & 1 Aneurysm & 3.0 & Yes & Yes/Yes & 0 & 9 & 6 \\
\hline
\end{tabular}

Note: *Spetzler-Martin grading scale; SAH, subarachnoid hemorrhage; NIHSS, National Institutes of Health Stroke 